\title{
Utility of Data Fusion for Public Health Monitors: Lessons Learned from a Beta Test
}

\author{
Rhonda A. Lizewski*1, Howard Burkom², Joseph Lombardo², Christopher Cuellar², \\ Yevgeniy Elbert ${ }^{2}$, Liane Ramac-thomas ${ }^{2}$ and Julie Pavlin ${ }^{1}$
}

${ }^{1}$ Armed Forces Health Surveillance Center, Silver Spring, MD, USA; ${ }^{2} J o h n s$ Hopkins Applied Physics Laboratory, Laurel, MD, USA

\section{Objective}

To evaluate, prior to launch, a surveillance system upgrade allowing analytical combination of weighted clinical and syndromic evidence with multiple severity indicators.

\section{Introduction}

The Armed Forces Health Surveillance Center (AFHSC) supports the development of new analytical tools to improve alerting in the Electronic Surveillance System for the Early Notification of Community-based Epidemics (ESSENCE) disease-monitoring application used by the Department of Defense (DoD). Developers at the Johns Hopkins University Applied Physics Laboratory (JHU/APL) have added an analytic capability to alert the user when corroborating evidence exists across syndromic and clinical data streams including laboratory tests and filled prescription records. In addition, AFHSC epidemiologists have guided the addition of data streams related to case severity for monitoring of events expected to require expanded medical resources. Evaluation of the multi-level fusion capability for both accuracy and utility is a challenge that requires feedback from the user community before implementation and deployment so that changes to the design can be made to save both time and money. The current effort describes the design and results of a large evaluation exercise.

\section{Methods}

We held a 2-day workshop on 14-15 May 2013 with attendees from public health communities in the Army, Navy and Air Force, to include ESSENCE users at local levels (responsible for one military installation) and at regional levels (responsible for an entire military Service or the entire DoD). The workshop was preceded by a webinar to introduce the fusion evaluation project and provide instruction on the prototype evaluation site prior to the workshop. Attendees used the ESSENCE demonstration site and evaluated the system using historical DoD events both as a group and individually. Workshop attendees discussed their experiences and suggestions for improvement through interactive moderated sessions and Groupware software, an instant messaging type service which allowed comments to be made and shared with the group outside of the discussion. In addition, attendees completed structured surveys after each scenario to obtain feedback.

\section{Results}

Respondents unanimously affirmed the overall utility of the fusion capability. A strong majority asserted that the fusion capability would give confidence for follow-up investigations and favored initial use followed by investigation of single-stream alerts. However, concerns about the visualization and lack of detail for some data sources caused reservations. Multiple respondents noted that utility of the system is role-specific with local users wanting agility between combined fusion and single-stream information and regional users using fusion to supplement lack of knowledge of local events. For importance of data sources, users most often chose severity information as useful, with some stressing clinical data, and one interested strictly in timeliness.

\section{Conclusions}

Both the need and the approach for this analytic fusion capability were approved, with comments dependent on the background and jurisdiction of the participant. Attendees made suggestions in the general areas of improving severity data, algorithms, and the fusion system. For severity-related indicators, they recommended 1) not using "acute" appointment types as these are often used by treatment facilities for any new appointment, 2) using disposition codes for "sick-in-quarters" in addition to hospitalization as an indicator of more severe illness, 3) filtering complex management codes for increased specificity, and 4) not including second appointments coded "routine" in the bounce-back category. For improving algorithms, they recommended 1) monitoring ratios of positive influenza tests, 2) monitoring both young and older age distributions as a separate indicator, and 3 ) adjusting algorithms for total facility visit counts. For improvements to the fusion system, they recommended 1) better representation of data streams, 2) modifying the severity fusion process to prioritize new data streams and 3) cross-validating the improved network on the beta test events. In addition, attendees provided multiple suggestions on the graphical user interface to improve utility of the system.

\section{Keywords}

Analytic; ESSENCE; Fusion

\section{*Rhonda A. Lizewski}

E-mail: rhonda.a.lizewski.mil@mail.mil 